\title{
Innovative web-based tool for promoting well-being among healthy adolescents: An implementation protocol
}

\author{
Trivellini $\mathrm{G}^{1 *}$, Doveri $\mathrm{C}^{1 \neq}$, Mastorci $\mathrm{F}^{1}$, Bastiani $\mathrm{L}^{1}$, Cappa $\mathrm{C}^{1}$, Vassalle $\mathrm{C}^{2}$ and Pingitore $\mathrm{A}^{1 *}$ \\ ${ }^{1}$ Clinical Physiology Institute, CNR, Pisa, Italy \\ ${ }^{2}$ Fondazione G. Monasterio, Regione Toscana, Pisa, Italy \\ \#Contributed equally to this work
}

\begin{abstract}
The purpose of this article is to develop a new Web-based tool in order to assess lifestyle habits (L), social context (S), emotional status (E), and mental skills (M) in adolescents, through an integrated index of the best indicators of well- being.

This Web-tool generates a personalized well-being index (PWBI). A pilot study, conducted on 291 healthy adolescents, has been set up, to define the feasibility of the platform, from sampling via Web-tool to data processing. The estimated values of the four well-being dimension weights, for one of 291 students as example, are $\mathrm{L}=0.590, \mathrm{E}=0.851, \mathrm{~S}=0.342$ and $\mathrm{M}=0.943$. By normalizing their sum to unity, the relative impacts on the total $\mathrm{PWBI}$ of the $\mathrm{L}, \mathrm{E}, \mathrm{S}$, and $\mathrm{M}$ are $22 \%, 31 \%, 12 \%$ and $35 \%$ respectively.

The Web-platform proposed here may represent a feasible and flexible tool for health and well-being management of adolescents from epidemiological, preventive, and educational point of views.
\end{abstract}

\section{Introduction}

\section{Background}

Adolescence is a challenging period of the life course characterized by cognitive, physical, psychological and emotional changes, which impacts on health and wellbeing [1]. About half of the adult disease are estimated to have their onset in the early adolescence [2], with profound implications for social development and global economy, making the early adolescence particularly pivotal for implementing interventions. To date, preventive strategies, mainly regarding food, nutrients intake and physical activity, are directed towards adolescents with mental disorders or physical illness rather than the healthy ones. However, preventive interventions in healthy adolescent population is useful to improve resilience, happiness, social involvement, selfesteem and sociability, in order to reduce potential risk factors. This is in accord with the World Health Organization health definition that is "a state of well-being, in which the individual realizes his or her own abilities, can cope with the normal stresses of life, can work productively and fruitfully, and is able to make a contribution to his or her community". Accordingly, the spectrum of risk factors ranges from purely genetic to behavioural, psychosocial and environmental ones, creating an integrated and complex causal pathway, key when applying preventive strategies to improve well-being. Furthermore, adolescent population often represents a challenge in the prevention field as teens begin the transition process of parent-managed healthcare to personal responsibility for health behaviour and well-being.

In this framework, the new era of computer technology is actually pervasive in healthcare and, in particular, Web tools provide the opportunity to gather data in a fast, cheap and low time-consuming way, favouring also the possibility to integrate the information each other [3].

\section{Objectives}

The aim of this paper is to present a new Web-based tool, with the following goals: 1) to create a new innovative tool to assess lifestyle habits, social context, emotional status, and mental skills in adolescents, through an integrated index of the best indicators of well-being; 2 ) to design a Web-based tool for the improvement of the adolescents' wellbeing through a dynamic and personalized interface which provides an immediate automated feedback that will allow monitoring progresses; 3) to develop a powerful tool from societal, cultural, epidemiological, economic and environmental perspectives, directed towards adolescents, teachers, and parents.

\section{Methods}

\section{General aspects of the Web-tool}

The platform was designed to collect data on fundamental aspects of adolescent daily life, free use of charge, without commercial license, by the scholastic community (scholars, teachers, parents). Development of the project included the following steps: 1) lifestyle habits, social context, emotional status, and mental skills identification model; 2) execution of a pilot study on a limited set of adolescents enrolled in Italian schools to verify the feasibility of the platform; 3 ) modification and development of the Web-platform, if necessary, based on evidence raised by the pilot study; 4) distribution of the Web-tool to Italian

${ }^{\star}$ Correspondence to: Alessandro Pingitore, Clinical Physiology Institute, CNR, Via Moruzzi, 1, 56124, Pisa, Italy, Tel: +39050312605, Email: pingi@ifc.cnr.it

Key words: adolescent, health management, technology, web-tool, well-being

Received: June 06, 2018; Accepted: June 25, 2018; Published: June 28, 2018 
school network. The first two steps have been completed, while step 3 and step 4 are now in progress.

\section{Dataset and measures}

The dataset, including lifestyle habits, social context, emotional status, and mental skills dimensions, was created by a multidisciplinary research group of specialists (medical doctors, biologists, computer technicians, epidemiologists, psychologists) to measure quality of life and well-being in adolescent population.

Traditional tools to gather information, via Web platform, about individual psychosocial measures include a well-known, validated, and standardized questionnaire, the KIDSCREEN-52 used for knowing the quality of life perception of the adolescent based on five scales: physical and psychological well-being, autonomy and parent relation, peers and social support, and school environment [4]. In addition, a battery of neuropsychological tests to evaluate a broad range of mental skills (attention and inhibitory control), has been implemented in the Web-tool. The questionnaire and cognitive test are collected online for a maximum of $60 \mathrm{~min}$ in a computer class with a teacher who presided over the data collection. The questionnaire is completed anonymously, voluntarily, and the study has been approved by the Institutional Ethical Review Board Committee.

\section{Structure and function of the Web-tool}

Interactive Web pages allow to the teachers, students, and parents to run the software and then store, retrieve, compute, and show student/ son's data. In this way, all data and software are located on the server alone, sited at the Clinical Physiology Institute, CNR (Pisa), and no special client software is needed. The only requirement for the user is to know the server Web address and to own a registered account.

The Web-tool platform offers:

- a framework for acquiring data over time

- a multimodal data processing module, with dedicated algorithms which extract a number of variables correlated with health status.

- a data fusion and synthesis module, to define an integrated and personalized Well-being Index. The index of the health status and wellbeing of an adolescent will be traced over time, thus enabling users to relate the adolescent profile to their well-being.

- a personalised user guidance module, which acts as a kind of wellbeing navigator to support users in the achievement and maintenance of a correct health status and well-being.

The platform is a Web application based on a client-server architecture developed entirely by using open source software. The back-end structure is made up of a Linux server and a MYSQL database backed up daily to ensure data loss recovery in the event of hardware or software issues.

The application has been implemented with the integration of the following software- Yii2 is a Web framework based on PHP language that leverages MVC architecture, integrating JavaScript, JQuery, and AJAX. Through the use of this framework, the user management, institute management, welfare index processing, data output, and integration with the following software have been developed.

- LimeSurvey (PHPSurveyor) is an application developed to generate online statistical questionnaires, which allows the user/ developer to intervene on the code (ID), to personalize and further develop it through the PHP language.
- Joomla is a CMS software platform (content management system) for Web site creation and optimization, written entirely in PHP language, and based on MYSQL database.

Users will be able to access the platform at any time using a browser from any device (smartphone, tablet, personal computer) through a username and login. The protocol used for server-to-client (browser) communications is SSL (Security Socket Layer) and is essential to ensure the confidentiality of data while using the platform by the users. Using an SSL certificate installed on the server identifies the legitimate recipient of the information sent by the user making them unreadable to everyone else because they are encrypted.

\section{Multimodal data processing}

The multimodal data acquired by the platform are processed to extract reliable measures linked to health status and well-being of adolescents. We have defined a list of best indicators of health status and well-being in order to outline an integrated index for each adolescent and to determine how each indicator represents and contributes to the underlying of this index.

The software enables the teacher, students, and parents to identify the student's well-being index through several computing and referring instruments, as listed below:

- KIDSCREEN questionnaire: based on physical, emotional, mental, social, and behavioural components of perceived well-being by adolescent.

- An integration of well-being determinants included in lifestyle habits, social context, emotional status, and mental skills domains. In particular, two statistical methods were developed: i) Cluster analysis, to classify a sample of adolescents (based on a set of measured variables) into a number of different groups; ii) Structural Equation Models (SEMs), to define a personalized well-being index (PWBI), and to determine how each of the variables considered (well-being determinants assessed by means of questionnaire) represent and contributes to the underlying latent construct of index (Figure 1). For each adolescent will be calculated the contribution (Weight) of the single question (item) in the measurement of the underlying latent constructs (Lifestyle Habits: Nutrition, Physical Activity, Sleep... etc.). The chance to identify the different behaviours in adolescents with the same score in the various latent variables (Low, Intermediate, High level) will allow to calibrate personalized interventions aimed to promote well-being.

\section{Results}

\section{Sample size calculation}

To determine optimal sample-size of this study we will refer to the frequently promoted $\mathrm{N}$ : $\mathrm{t}$ rule of thumb concerns the minimum recommended ratio of sample size $(\mathrm{N})$ to number of parameters $(\mathrm{t})$ to be estimated in a Structural Equation Models (SEM). Various N: $\mathrm{t}$ ratios were proposed in SEM literature, the typical 7:1 ratio: 5:1 ratio, but also the 3:1 and 2:1, was suggested [5]. Our conceptual SEM has about 100 parameters for the measurement model of the latent variables and 18 parameters for the path analysis model of the latent variable links. Thus, with $\mathrm{t}=116$, we estimated a minimal sample size of $118 \times 2$, or $\mathrm{N}=236$ for a $2: 1$ ratio (Pilot study, step 2 of the project) or $\mathrm{N}=354$ for a $3: 1$ ratio, or $\mathrm{N}=590$ for a 5:1 ratio, or $\mathrm{N}=826$ for $7: 1$ ratio (step 4 of the Project, now in progress). 


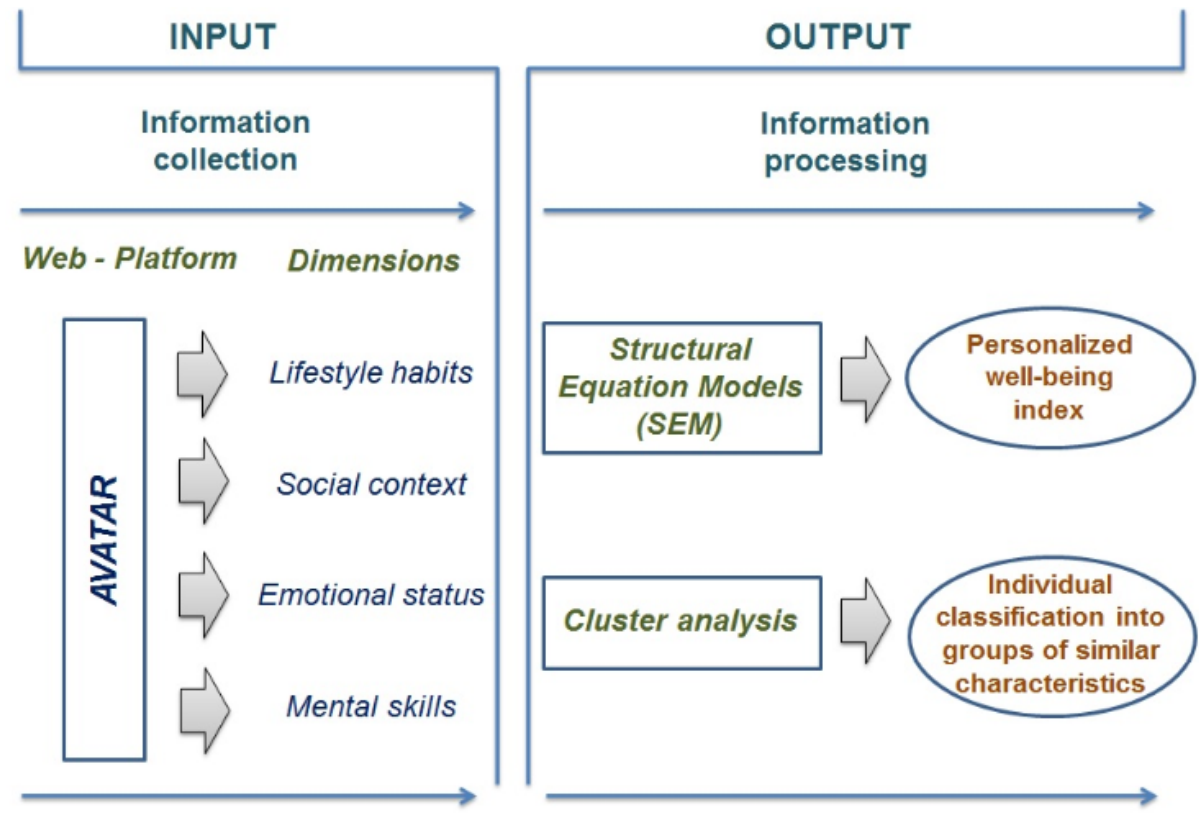

Figure 1. The schema of data fusion used to estimate the Personalized Well-Being Index (PWBI) and classify adolescents into a number of different groups

\section{Pilot study}

To verify the feasibility of the platform, and identify any glitches, from sampling via Web-tool to data processing, a sample of 291 (2:1 ratio) healthy adolescents (140 males), nationally representative, from five Italian schools (Tuscany, Liguria, Lombardy, Calabria) was enrolled.

Adolescents were eligible to participate if they were in the age range of 10-14 (inclusive) years, and read and speak Italian. Legal guardians gave informed consent, and authorized physicians to use data in accordance with Italian law. All procedures performed in the study were in accordance with the ethical standards of the institutional and/ or national research committee and with the 1964 Helsinki declaration and its later amendments or comparable ethical standards.

\section{Personalized Well-Being Index (PWBI)}

Both computed and user-provided data, obtained from the platform, can be mapped onto a specific axis of $\Sigma$ space. It follows that PWBI implementation is referred back to estimating four mappings,

Lifestyle habits (L): $\Sigma \mathrm{L} \rightarrow \Omega \mathrm{L}$

Social context (S): $\Sigma \mathrm{S} \rightarrow \Omega S$

Emotional status (E): $\Sigma \mathrm{E} \rightarrow \Omega \mathrm{E}$

Mental skills (M): $\rightarrow \Sigma \mathrm{M} \Omega \mathrm{M}$

between the predefined subareas $(\Sigma \mathrm{L}, \Sigma \mathrm{S}, \Sigma \mathrm{E}$ and $\Sigma \mathrm{M})$ of and the four axes (L, S, E, and M). Each of these causal relationships is modelled by a linear equation with the cause(s) as independent variable(s). Structural equation models (SEMs), widely used in psychometry and behavioural sciences, were used to implement the association phase [6]. This choice was motivated by the moderate complexity of a linear SEM, which despite a possible negative influence on the estimation accuracy, is advantageous with respect to overfitting issues.

\section{Preliminary results}

Of 291 students recruited at the start of school year, only 9 did not complete all items of the questionnaire. Technical glitches, such as trouble entering server, were observed in only $3 \%$ of cases.
These preliminary results allowed us to define the logarithm for the personalized well-being index. The estimated values of the four well-being dimension weights, for one of 291 students as example, are $\mathrm{L}=0.590, \mathrm{E}=0.851, \mathrm{~S}=0.342$ and $\mathrm{M}=0.943$. By normalizing their sum to unity, the relative impacts on the total PWBI of the Lifestyle, Emotional status, Social context, and Mental skill are 22\%, 31\%, 12\% and 35\% respectively.

In the same way, it is possible to calculate the contribution of each variable in the construction of the different dimensions (Figure 2).

\section{Discussion}

The implementation of the Web-platform based on a wide-range of data integration might represent a useful tool to improve adolescent's overall self-esteem, resilience, and self-empowerment.

Current preventive strategies are mainly directed towards adolescents with disorders rather than the healthy ones, even if healthy policies support the necessity of preventive interventions in healthy adolescent population before it is too late [7].

Despite this need, nowadays, several health and well-being education programs in adolescent people are focused on nutrition and physical activity fields, where it has been documented an effective raise awareness in increasing physical activity and changing nutrition behaviour [8]. To our knowledge, few studies have examined the use of Web-based tools and feedback for adolescent healthy behaviours outside of alcohol or substance abuse.

However, prevention strategies, in particular from a primordial prevention point of view, should not only aim at reducing lifestyle risk behaviours, but also improving well-being by increasing resilience, happiness, social involvement, self-esteem and sociability, which are all determinants of positive health status, and thus, higher quality of life. This requires a holistic developmental approach considering that each adolescent has different and changing needs depending on one's age, level of maturity and social context [9]. The Web-tool we are proposing has incorporated elements from international reports with measures of social role transitions, psychological profile, cognitive functions, and risk and protective factors. The concept underpinning the idea is not 


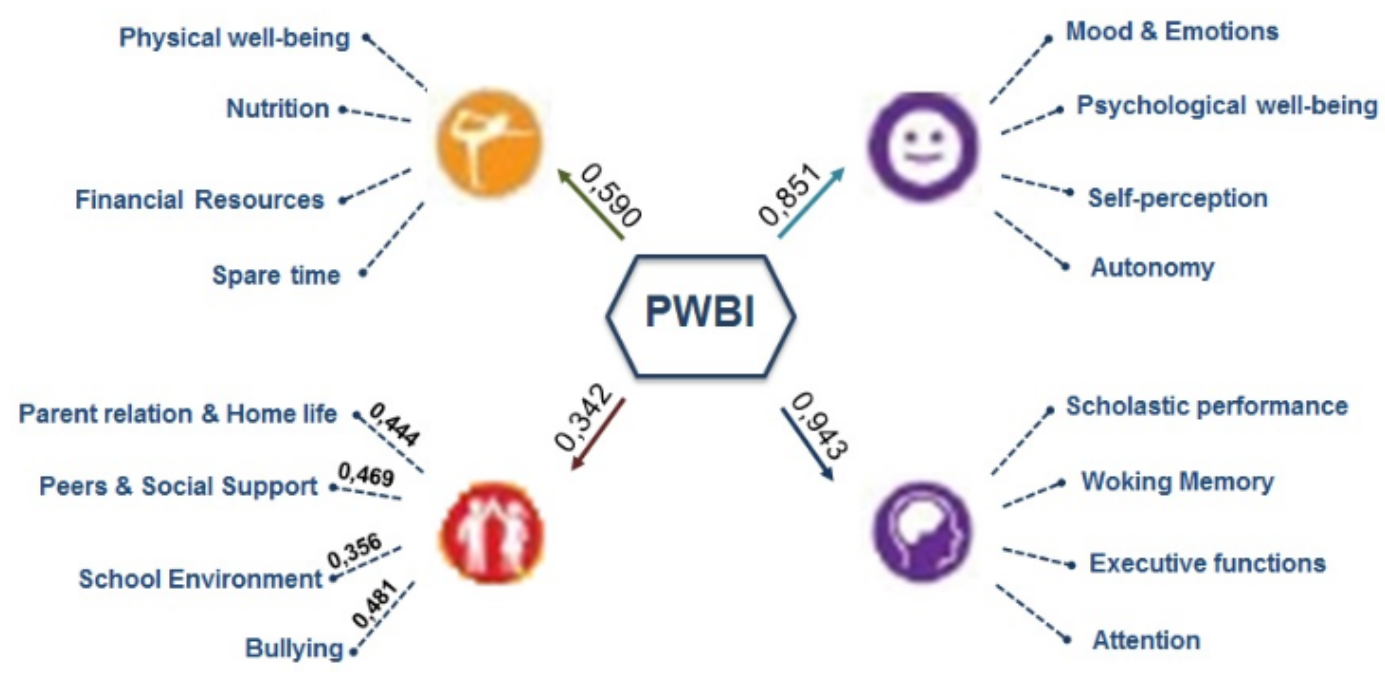

Figure 2. Graphical representation of the Personalized Well-Being Index (PWBI) for one student as example in the form in which it appears in the Web-tool. The PWBI is presented along with the lifestyle habits, social context, emotional status, and mental skills components

to produce a list of the best indicators of health status, but rather to outline an integrated index of well-being for each adolescent and to determine how each indicator (health determinants) contributes to this index. The development of a personalised preventive strategy plan, as recommended by the Web-tool, requires knowledge on adolescents' age-related stage from a physical, mental, and social point of view, and of their socio-cultural environment, including their interests, concerns and activities in their spare time. The tool is designed to achieve a Webbased health promotion platform for adolescents, parents, teachers, psychologists, and other stakeholders, with particular attention to school environment as "context of socialization" that influences student's developmental outcomes. The Web-tool, here described, intervenes at multiple points in the promotion process of health and well-being in the young, combining management and empowerment strategies with scholastic programmes and good practices for health promotion.

The Web-based approach, during pilot phase, has been used for research purposes, studying a number of adolescents and creating a comprehensive profile for the assessment of well-being based on psychological, cognitive, social, and environmental factors. The tool helps to define personalized preventive interventions, addressed to the community and to the individual, at-school and at-home.

From the point of view of platform design and creation, the tool was evaluated as very useful and user friendly by the three organizational levels (adolescents, teachers, and parents), comparing their experience to the previous scholastic programmes. When prompted with possible adaptations and developments to the tool, adolescents expressed interest and attention in receiving follow-up information about health status and well-being, opportunities to define goals and track well-being index during time. The dynamic engagement suggests that adolescents may envision an active role in health and well-being behaviours change thanks to the possibility to self-regulate unhealthy behaviours.

Taken together, adolescents' interest in highly personalized programs lends additional support to increasing evidence that Webbased interventions incorporating tailored information yield stronger effects than generic interventions that not consider the individual variability.
The major innovations of this Web-tool well-being assessment and promotion are the simplicity and usability data acquisition that is as comprehensive in terms of both well-known and less-known wellbeing indicators, including psychosocial and behavioural dimensions. The use of this multifactorial approach allows the three levels of stakeholders (students, teachers, parents) to automatically retrieve from the database data on adolescents' well-being. Teachers can directly visualize: the cluster of students with similar profiles based on the integrated view obtained from lifestyle habits, social context, mental skills, and emotional status (teachers), and the integrated personalized index of well-being of each teenager in the class. Parents can visualize the integrated personalized index of well-being of own child. Also, adolescents can visualize directly the own integrated personalized index of well-being.

The Web-tool is highly flexible and adaptable in its potential applications. This helps teachers to select more appropriately personalized interventions and educational programs for individual student and for the students of a classroom and, thus, monitor their compliance and effectiveness, by producing graphical trends of single student or class over time. In addition, the platform can be considered a data repository tool for epidemiological studies to archive reliable and comparable data of adolescents well-being determinants.

Finally, the development of the Web-platform, represents a powerful tool for the promotion of health and well-being in which a network of different stakeholders dedicated to the adolescence education may cooperate together to increase awareness, change behaviour, and create environments that support good health practices.

\section{Conclusion}

The Web-platform is a feasible and flexible tool for health and wellbeing management of adolescents from epidemiological, preventive, and educational point of views. A new algorithm based on multifactorial profile for the assessment of lifestyle habits, social context, mental skills and emotional status could be provided for the young people after collection of a significant amount of data by the present Web-based tool. Monitoring of lifestyle, social, emotional status and mental skills parameters through the platform will contribute to identify the role 
of each parameter in well-being index definition, and thus, to create preventive and personalized interventional strategies.

\section{Conflicts of interest}

The authors declare no conflict of interest.

\section{Acknowledgment}

The authors acknowledge the following Italian schools, that join the Italian school network RETE ULISSE, Scuole insieme per la ricerca scientifica e l'innovazione didattica:

1. Istituto Comprensivo Statale L. Strenta Tongiorgi, Pisa, Toscana

2. Istituto Comprensivo P Aldi, Manciano, Grosseto, Toscana

3. Istituto Comprensivo Il Milione, Suzzarra, Mantova, Lombardia

4. Istituto Comprensivo Carazze-Celle, Savona, Liguria

5. Istituto Comprensivo Statale Tiriolo, Catanzaro, Calabria.

They were fundamental to aquire data of the students.

The authors received no financial support for the research, authorship, and/or publication of this article.

\section{References}

1. McLaughlin KA, Garrad MC, Somerville LH (2015) What develops during emotional development? A component process approach to identifying sources of psychopathology risk in adolescence. Dialogues Clin Neurosci 17: 403-410. [Crossref]

2. Kessler RC, Avenevoli S, McLaughlin KA (2012) Lifetime co-morbidity of DSM-IV disorders in the US National Comorbidity Survey Replication Adolescent Supplement (NCS-A). Psychol Med 42: 1997-2010. [Crossref]

3. Buzzi MC, Buzzi M, Franchi D (2017) Facebook: a new tool for collecting health data? Multimedia Tools and Applications 76: 10677-10700.

4. Ravens-Sieberer U, Herdman M, Devine J (2014) The European KIDSCREEN approach to measure quality of life and well-being in children: development, current application, and future advances. Qual Life Res 23: 791-803.

5. Bagozzi RP and Yi Y (2012) Specification, evaluation, and interpretation of structural equation models. J of the Acad Mark Sci 40: 8-34.

6. Andreu Y, Chiarugi F, Colantonio S (2016) Wize Mirror - a smart, multisensory cardiometabolic risk monitoring system. Computer Vision and Image Understanding 148: $3-22$

7. Patton GC, Sawyer SM, Santelli JS (2016) Our future: a Lancet commission on adolescent health and wellbeing. Lancet 387: 2423-2478.

8. de Vries H, Kremers SP, Smeets T (2008) The effectiveness of tailored feedback and action plans in an intervention addressing multiple health behaviors. Am J Health Promot 22: 417-425. [Crossref]

9. Remschmidt H, Belfer M (2005) Mental health care for children and adolescents worldwide: a review. World Psychiatry 4: 147-153. [Crossref]

Copyright: (C2018 Trivellini G. This is an open-access article distributed under the terms of the Creative Commons Attribution License, which permits unrestricted use, distribution, and reproduction in any medium, provided the original author and source are credited. 